\title{
Analysis of Arithmetic Coding for Data Compression
}

\author{
Paul G. Howard and Jeffrey Scott Vitter
}

Brown University

Department of Computer Science

Technical Report No. CS-92-17

Revised version, April 1992

(Formerly Technical Report No. CS-91-03)

Appears in Information Processing and Management, Volume 28, Number 6, November 1992, pages 749-763.

A shortened version appears in the proceedings of the IEEE Computer Society/NASA/CESDIS Data Compression Conference, Snowbird, Utah, April 8-11, 1991, pages 3-12. 



\title{
Analysis of ARIthmetic Coding FOR DATA COMPRESSION ${ }^{1}$
}

\author{
Paul G. Howard ${ }^{2} \quad$ Jeffrey Scott Vitter $^{3}$ \\ Department of Computer Science \\ Brown University \\ Providence, R.I. 02912-1910
}

\begin{abstract}
Arithmetic coding, in conjunction with a suitable probabilistic model, can provide nearly optimal data compression. In this article we analyze the effect that the model and the particular implementation of arithmetic coding have on the code length obtained. Periodic scaling is often used in arithmetic coding implementations to reduce time and storage requirements; it also introduces a recency effect which can further affect compression. Our main contribution is introducing the concept of weighted entropy and using it to characterize in an elegant way the effect that periodic scaling has on the code length. We explain why and by how much scaling increases the code length for files with a homogeneous distribution of symbols, and we characterize the reduction in code length due to scaling for files exhibiting locality of reference. We also give a rigorous proof that the coding effects of rounding scaled weights, using integer arithmetic, and encoding end-of-file are negligible.
\end{abstract}

Index terms: Data compression, arithmetic coding, analysis of algorithms, adaptive modeling.

\footnotetext{
${ }^{1}$ A similar version of this paper appears in Information Processing and Management, 28:6, November 1992, 749-763. A shortened version of this paper appears in the proceedings of the IEEE Computer Society/NASA/CESDIS Data Compression Conference, Snowbird, Utah, April 8-11, 1991, $3-12$.

${ }^{2}$ Support was provided in part by NASA Graduate Student Researchers Program grant NGT50420 and by an NSF Presidential Young Investigators Award with matching funds from IBM. Additional support was provided by a Universities Space Research Association/CESDIS appointment.

${ }^{3}$ Support was provided in part by a National Science Foundation Presidential Young Investigator Award grant with matching funds from IBM, by NSF grant CCR-9007851, by Army Research Office grant DAAL03-91-G-0035, and by the Office of Naval Research and the Defense Advanced Research Projects Agency under contract N00014-83-J-4052, ARPA order 8225. Additional support was provided by a Universities Space Research Association/CESDIS associate membership.
} 



\section{Introduction}

We analyze the amount of compression possible when arithmetic coding is used for text compression in conjunction with various input models. Arithmetic coding is a technique for statistical lossless encoding. It can be thought of as a generalization of Huffman coding [14] in which probabilities are not constrained to be integral powers of 2 , and code lengths need not be integers.

The basic algorithm for encoding using arithmetic coding works as follows:

1. We begin with a "current interval" initialized to [0..1].

2. For each symbol of the file, we do two steps:

(a) We subdivide the current interval into subintervals, one for each possible alphabet symbol. The size of a symbol's subinterval is proportional to the estimated probability that the symbol will be the next symbol in the file, according to the input model.

(b) We select the subinterval corresponding to the symbol that actually occurs next in the file, and make it the new current interval.

3. We transmit enough bits to distinguish the final current interval from all other possible final intervals.

The length of the final subinterval is clearly equal to the product of the probabilities of the individual symbols, which is the probability $p$ of the particular sequence of symbols in the file. The final step uses almost exactly $-\lg p$ bits to distinguish the file from all other possible files. For detailed descriptions of arithmetic coding, see [17] and especially [34].

We use the following notation throughout this article:

$$
\begin{aligned}
t & =\text { length of the file, in bytes; } \\
n & =\text { number of symbols in the input alphabet; } \\
k & =\text { number of different alphabet symbols that occur in the file; } \\
c_{i} & =\text { number of occurrences of the } i \text { th alphabet symbol in the file; } \\
\lg x & =\log _{2} x ; \\
A^{\bar{B}} & =A(A+1) \cdots(A+B-1) \quad \text { (the rising factorial function). }
\end{aligned}
$$

Results for a "typical file" refer to a file with $t=100,000, n=256$, and $k=100$. Code lengths are expressed in bits. We assume 8-bit bytes.

\subsection{Modeling effects}

The coder in an arithmetic coding system must work in conjunction with a model that produces probability information, describing a file as a sequence of decisions; at 
each decision point it estimates the probability of each possible choice. The coder then uses the set of probabilities to encode the actual decision.

To ensure decodability, the model may use only information known by both the encoder and the decoder. Otherwise there are no restrictions on the model; in particular, it can change as the file is being encoded. In this subsection we describe several typical models for context-independent text compression.

The length of the encoded file depends on the model and how it is used. Most models for text compression involve estimating the probability $p$ of a symbol by

$$
p=\frac{\text { weight of symbol }}{\text { total weight of all symbols }} \text {, }
$$

which we can then encode in $-\lg p$ bits using exact arithmetic coding. The probability estimation can be done adaptively (dynamically estimating the probability of each symbol based on all symbols that precede it), semi-adaptively (using a preliminary pass of the input file to gather statistics), or non-adaptively (using fixed probabilities for all files). Non-adaptive models are not very interesting, since their effectiveness depends only on how well their probabilities happen to match the statistics of the file being encoded; Bell, Cleary, and Witten show that the match can be arbitrarily bad $[2]$.

Static and decrementing semi-adaptive codes. Semi-adaptive codes are conceptually simple, and useful when real-time operation is not required; their main drawback is that they require knowledge of the file statistics prior to encoding. The statistics collected during the first pass are normally used in a static code; that is, the probabilities used to encode the file remain the same during the entire encoding. It is possible to obtain better compression by using a decrementing code, dynamically adjusting the probabilities to reflect the statistics of just that part of the file not yet coded.

Assuming that encoding uses exact arithmetic, and that there are no computational artifacts, we can use the file statistics to form a static probabilistic model. Not including the cost of transmitting the model, the code length $L_{S S}$ for a static semi-adaptive code is

$$
\begin{aligned}
L_{S S} & =-\lg \prod_{i=1}^{n}\left(c_{i} / t\right)^{c_{i}} \\
& =t \lg t-\sum_{i=1}^{n} c_{i} \lg c_{i},
\end{aligned}
$$

the information content of the file. Dividing by the file length gives the self-entropy of the file, and forms the basis for claims that arithmetic coding encodes asymptotically close to the entropy. What these claims really mean is that arithmetic coding can encode close to the entropy of a probabilistic model whose symbol probabilities are 
the same as those of the file, because computational effects (discussed in Section 3) are insignificant.

If we know a file's exact statistics ahead of time, we can get improved compression by using a decrementing code. We modify the symbol weights dynamically (by decrementing each symbol's weight each time it is encoded) to reflect symbol frequencies for just that part of the file not yet encoded; hence for each symbol we always use the best available estimates of the next-symbol probabilities. In a sense, it is a misnomer to call this a "semi-adaptive" model since the model adapts throughout the second pass, but we apply the term here since the symbol probability estimates are based primarily on the first pass. The decrementing count idea appears in the analysis of enumerative codes by Cleary and Witten [7]. The resulting code length for a semi-adaptive decrementing code is

$$
L_{S D}=-\lg \left(\left(\prod_{i=1}^{k} c_{i} !\right) / t !\right) .
$$

It is straightforward to show that for all input files, the code length of a semiadaptive decrementing code is at most that of a semi-adaptive static code, equality holding only when the file consists of repeated occurrences of a single letter. This does not contradict Shannon's theorem [30]; he discusses only the best static code.

Static semi-adaptive codes have been widely used in conjunction with Huffman coding, where they are appropriate since changing weights often requires changing the structure of the coding tree.

Encoding the model. If we assume that all symbol distributions are equally likely for a given file length $t$, the cost of transmitting the exact model statistics is

$$
\begin{aligned}
L_{M} & =\lg (\text { number of possible distributions }) \\
& =\lg \left(\begin{array}{c}
t+n-1 \\
n-1
\end{array}\right) \\
& \sim n \lg (\text { et } / n) .
\end{aligned}
$$

A similar result appears in [2] and [7]. For a typical file $L_{M}$ is only about 2560 bits, or 320 bytes. The assumption of equally-likely distributions is not very good for text files; in practice we can reduce the cost of encoding the model by 50 percent or more by encoding each of the counts using a suitable encoding of the integers, such as Fibonacci coding [12].

Strictly speaking we must also encode the file length $t$ before encoding the model; the cost is insignificant, between $\lg t$ and $2 \lg t$ bits using an appropriate encoding of integers $[11,31,32]$.

Adaptive codes. Adaptive codes use a continually changing model, in which we use the frequency of each symbol up to a given point in the file as an estimate of its probability at that point. 
We can encode a symbol using arithmetic coding only if its probability is nonzero, but in an adaptive code we have no way of estimating the probability of a symbol before it has occurred for the first time. This is the zero-frequency problem, discussed at length in $[1,2,33]$. For large files with small alphabets and simple models, all solutions to this problem give roughly the same compression. In this section we adopt the solution used in [34], simply assigning an initial weight of 1 to all alphabet symbols. The code length using this adaptive code is

$$
L_{A}=-\lg \left(\left(\prod_{i=1}^{k} c_{i} !\right) / n^{\bar{t}}\right) .
$$

By combining Equations (1), (2), and (3) and noting that $\left(\begin{array}{c}t+n-1 \\ n-1\end{array}\right)=n^{\bar{t}} / t$ !, we can show that for all input files, the adaptive code with initial 1-weights gives the same code length as the semi-adaptive decrementing code in which the input model is encoded based on the assumption that all symbol distributions are equally likely. In other words, $L_{A}=L_{S D}+L_{M}$. This result is found in Rissanen [23,24]. Cleary and Witten [7] and Bell, Cleary, and Witten [2] present a similar result in a more general setting, showing approximate equality between enumerative codes (which are similar to arithmetic codes) and adaptive codes. Intuitively, the reason for the equality is that in the adaptive code, the cost of "learning" the model is not avoided, but merely spread over the entire file [25].

Organization of this article. Section 2 contains our main result, which precisely and provably characterizes the code length of a file dynamically coded with periodic count-scaling. We express the code length in terms of "weighted entropies" of the model, which are the entropies implied by the model at the scaling times. Our result shows both the advantage to be gained by scaling because of a locality-of-reference effect and the excess code length incurred by the overhead of the scaling process. For example, scaling has the most negative effect when the alphabet symbols are distributed homogeneously throughout the file, and our result shows explicitly the small amount that scaling can cause the code length to increase. However, when the distribution of the alphabet symbols varies, as is often the case in files displaying locality of reference, our result characterizes precisely the benefits of scaling on code length. In Section 2.2 we extend this analysis to higher-order models based on the partial string matching algorithm of Cleary and Witten.

Through the years, practical adjustments have been made to arithmetic coding [21, $26,28,34]$ to allow the use of integer rather than rational or floating point arithmetic and to transmit output bits almost in real time instead of all at the end. In Section 3 we prove that the loss of coding efficiency caused by practical coding requirements is negligible, thus demonstrating rigorously the empirical claims made in $[2,34]$. 


\section{Scaling}

Scaling is the process in which we periodically reduce the weights of all symbols. It allows us to use lower precision arithmetic at the expense of making the model more approximate, which can hurt compression when the distribution of symbols in the file is fairly homogeneous. Scaling also introduces a locality of reference (or recency) effect, which often improves compression when the distribution of symbols is variable. In this section we give a precise characterization of the effect of scaling on code length produced by an adaptive model. We express the code length in terms of the weighted entropies of the model. The weighted entropy is the Shannon entropy, computed using probabilities weighted according to the scaling process; the term "weighted entropy" is a notational convenience. Our characterization explains why and by how much scaling can hurt or help compression.

In most text files we find that most of the occurrences of at least some words are clustered in one part of the file. We can take advantage of this locality by assigning more weight to recent occurrences of a symbol in a dynamic model. In practice there are several ways to do this:

- Periodically restarting the model. This often discards too much information to be effective, although Cormack and Horspool find that it gives good results when growing large dynamic Markov models [8].

- Using a sliding window on the text [15]. This requires excessive computational resources.

- Recency rank coding $[4,10,29]$. This is computationally simple but corresponds to a rather coarse model of recency.

- Exponential aging (giving exponentially increasing weights to successive symbols) $[9,20]$. This is moderately difficult to implement because of the changing weight increments.

- Periodic scaling [34]. This is simple to implement, fast and effective in operation, and amenable to analysis. It also has the computationally desirable property of keeping the symbol weights small. In effect, scaling is a practical version of exponential aging. This is the method that we analyze.

In our discussion of scaling, we assume that a file is divided into blocks of length $B$. Our model assumes that at the end of each block, the weights for all symbols are multiplied by a scaling factor $f$, usually $1 / 2$. Within a block we update the symbol weights by adding 1 for each occurrence.

Example 1: We illustrate an adaptive code with scaling, encoding the 8-symbol file "abacbcba". In this example, we start with counts $c_{a}=1, c_{b}=1$, and $c_{c}=1$, and set

the scaling threshold at 10 , so we scale only once, just before the last symbol in the 
file. To retain integer weights without allowing any weight to fall to 0 , we round all fractional weights obtained during scaling to the next higher integer.

\begin{tabular}{c|cc|ccc} 
Symbol & \multicolumn{2}{|c|}{ Current Interval } & $p_{a}$ & $p_{b}$ & $p_{c}$ \\
\hline \hline Start & 0.000000000 & 1.000000000 & $1 / 3$ & $1 / 3$ & $1 / 3$ \\
\hline$a$ & 0.000000000 & 0.333333333 & $2 / 4$ & $1 / 4$ & $1 / 4$ \\
$b$ & 0.166666667 & 0.250000000 & $2 / 5$ & $2 / 5$ & $1 / 5$ \\
$a$ & 0.166666667 & 0.200000000 & $3 / 6$ & $2 / 6$ & $1 / 6$ \\
$c$ & 0.194444444 & 0.200000000 & $3 / 7$ & $2 / 7$ & $2 / 7$ \\
$b$ & 0.196825397 & 0.198412698 & $3 / 8$ & $3 / 8$ & $2 / 8$ \\
$c$ & 0.198015873 & 0.198412698 & $3 / 9$ & $3 / 9$ & $3 / 9$ \\
$b$ & 0.198148148 & 0.198280423 & $3 / 10$ & $4 / 10$ & $3 / 10$ \\
\hline Scaling & & & $2 / 6$ & $2 / 6$ & $2 / 6$ \\
\hline$a$ & 0.198148148 & 0.198192240 & $3 / 7$ & $2 / 7$ & $2 / 7$
\end{tabular}

The final interval is $[0.00110010101110011101,0.00110010101111001011]$ in binary. The theoretical code length is $-\lg 0.000044092=14.469$ bits. The actual code length is 15 bits, since the final subinterval can be determined from the output 001100101011101.

In this section we assume an adaptive model and exact rational arithmetic. We introduce the following additional notation for the analysis of scaling:

$$
\begin{aligned}
a_{i} & =\text { the } i \text { th alphabet symbol that occurs in the block; } \\
s_{i} & =\text { weight of symbol } a_{i} \text { at the start of the block; } \\
c_{i} & =\text { number of occurrences of symbol } a_{i} \text { in the block; } \\
A & =\sum_{i=1}^{n} s_{i} \quad \text { (the total weight at the start of the block); } \\
B & =\sum_{i=1}^{n} c_{i} \quad \text { (the size of the block); } \\
C & =A+B \quad \text { (the total weight at the end of the block); } \\
f & =A / C \quad \text { (the scaling factor); } \\
q_{i} & =s_{i} / A \quad \text { (probability of symbol } a_{i} \text { at the start of the block); } \\
r_{i} & =\left(s_{i}+c_{i}\right) / C \quad \text { (probability of symbol } a_{i} \text { at the end of the block); } \\
b & =\text { number of blocks resulting from scaling; } \\
m & =\text { the minimum weight allowed for any symbol, usually } 1 .
\end{aligned}
$$

When $f=1 / 2$, we scale by halving all weights every $B$ symbols, so $A=B$ and $b \approx t / B$. In a typical implementation, $A=B=8192$. 


\subsection{Coding Theorem for Scaling}

In our scaling model each symbol's counts are multiplied by $f$ whenever scaling takes place. Thus scaling has the effect of giving more weight to more recent symbols. If we denote the number of occurrences of symbol $a_{i}$ in block $m$ by $c_{i, m}$, the weight $w_{i, m}$ of symbol $a_{i}$ after $m$ blocks is given by

$$
\begin{aligned}
w_{i, m} & =c_{i, m}+f w_{i, m-1} \\
& =c_{i, m}+f c_{i, m-1}+f^{2} c_{i, m-2}+\ldots
\end{aligned}
$$

The weighted probability $p_{i, m}$ of symbol $a_{i}$ at the end of block $m$ is then

$$
p_{i, m}=\frac{w_{i, m}}{\sum_{i=1}^{n} w_{i, m}} .
$$

We now define a weighted entropy in terms of the weighted probabilities:

Definition 1 The weighted entropy of a file at the end of the $m$ th block, denoted by $H_{m}$, is the entropy implied by the probability distribution at that time, computed according to the scaling model. That is,

$$
H_{m}=\sum_{i=1}^{n}-p_{i, m} \lg p_{i, m},
$$

where the $p_{i, m}$ are given by Equation (5).

We find that $l_{m}$, the average code length per symbol for block $m$, is related to the starting weighted entropy $H_{m-1}$ and the ending weighted entropy $H_{m}$ in a particularly simple way:

$$
\begin{aligned}
l_{m} & \approx \frac{1}{1-f} H_{m}-\frac{f}{1-f} H_{m-1} \\
& =H_{m}+\frac{f}{1-f}\left(H_{m}-H_{m-1}\right) .
\end{aligned}
$$

Letting $f=1 / 2$, we obtain

$$
l_{m} \approx 2 H_{m}-H_{m-1} .
$$

When we multiply by the block length and sum over all blocks, we obtain the following precise and elegant characterization of the code length in terms of weighted entropies:

Theorem 1 Let $L$ be the code length of a file compressed with arithmetic coding using an adaptive model with scaling. Then

$$
\begin{aligned}
& B\left(\left(\sum_{m=1}^{b} H_{m}\right)+H_{b}-H_{0}\right)-t \frac{k}{B} \\
& \quad<L<B\left(\left(\sum_{m=1}^{b} H_{m}\right)+H_{b}-H_{0}\right)+t\left(\frac{k}{B} \lg \left(\frac{B}{k_{\min }}\right)+O\left(\frac{k^{2}}{B^{2}}\right)\right)
\end{aligned}
$$


where $H_{0}=\lg n$ is the entropy of the initial model, $H_{m}$ is the (weighted) entropy implied by the scaling model's probability distribution at the end of block $m$, and $k_{\min }$ is the smallest number of different symbols that occur in any block.

This theorem enables us to compute the code length of a file coded with periodic scaling. To do the calculation we need to know only the symbol counts within each scaling block; we can then use Equations (4) and (5) and Definition 1. The occurrence of entropy-like terms in the code length is to be expected; the main contribution of Theorem 1 is to show the precise and simple form that the entropy expressions take.

\subsubsection{Proof of the upper bound}

We prove the upper bound of Theorem 1 by showing first that the code length of a block depends only on the beginning weights and block counts of the symbols, and not on the order of symbols within the block. Then we show that there is an order such that for each symbol certain equalities and inequalities hold, which we use to compute a value and an upper bound for the code length of all occurrences of a single symbol in one block. Finally we sum over all symbols to obtain the worst case code length of a block, and over all blocks to obtain the code length of the file. The proof of the lower bound is similar.

The first lemma enables us to choose any convenient symbol order within a block.

Lemma 1 The code length of a block depends only on the beginning weights and block counts of the symbols, and not on the order of symbols within the block.

Proof: The exact code length $L$ of a block,

$$
L=-\lg \left(\left(\prod_{i=1}^{k} s_{i}^{\overline{c_{i}}}\right) / A^{\bar{B}}\right),
$$

has no dependence on the order of symbols. For each symbol $a_{i}$, the adaptive model ensures that the numerators in the set of probabilities used for coding the symbol in the block always form the sequence $\left\langle s_{i}, s_{i}+1, \ldots, s_{i}+c_{i}-1\right\rangle$, and that for the block as a whole the denominators always form the sequence $\langle A, A+1, \ldots, A+B-1\rangle$.

In the next lemma we prove the almost obvious fact that there is some order of symbols such that the occurrences of each symbol are roughly evenly distributed through the block. This order will enable us to use a smoothly varying function to estimate the probabilities used for coding the occurrences.

Definition 2 A block of length $B$ containing $c_{1}, c_{2}, \ldots, c_{k}$ occurrences of symbols $a_{1}, a_{2}, \ldots, a_{k}$, respectively, has the evenly-distributed (or ED) property if for each symbol $a_{i}$ and for all $m, 1 \leq m \leq c_{i}$, the symbol occurs for the $m$ th time not after position $m B / c_{i}$. 
Lemma 2 Every distribution of symbol counts has an order with the ED property.

Proof: Let $r_{i}(j)$ be the number of occurrences of symbol $a_{i}$ required up through the $j$ th position in any ED order. Such an order exists if and only if $\sum_{i=1}^{k} r_{i}(j) \leq j$ for $1 \leq j \leq B$. We find that $r_{i}(j)=\left\lceil\frac{c_{i}(j+1)}{B}\right\rceil-1<c_{i}(j+1) / B$. Since $\sum_{i=1}^{k} c_{i}=B$, $\sum_{i=1}^{k} r_{i}(j)<j+1$, or $\sum_{i=1}^{k} r_{i}(j) \leq j$ since $\sum_{i=1}^{k} r_{i}(j)$ is an integer. This holds for any $j$, so an ED order exists.

Next we define a number of related sums and integrals approximating $l$, the code length of all occurrences of one symbol in one block. For a given symbol with probability $p=c / B$ within the block, we can divide the block into $c$ subblocks each of length $B / c=1 / p$. Then $p_{B}(k)$ and $p_{E}(k)$ give the probability that the dynamic model would give to the $k$ th occurrence of the symbol if it occurred precisely at the beginning and end of the $k$ th subblock respectively.

$$
p_{B}(k)=\frac{q A+k}{A+k / p} ; \quad p_{E}(k)=\frac{q A+k}{A+(k+1) / p} .
$$

The expressions $S_{L}$ and $S_{U}$ are the lower and upper bounds of the symbol's code length, based on its occurrences being as early or as late as possible in the subblocks.

$$
S_{L}=\sum_{j=0}^{c-1}-\lg p_{B}(j) ; \quad S_{U}=\sum_{j=0}^{c-1}-\lg p_{E}(j) .
$$

The integrals $I_{L}$ and $I_{U}$ approximate $S_{L}$ and $S_{U}$.

$$
I_{L}=\int_{0}^{c}-\lg p_{B}(x) d x ; \quad I_{U}=\int_{0}^{c}-\lg p_{E}(x) d x .
$$

We define $\Delta_{I}$ to be $I_{U}-I_{L}$. After a considerable amount of algebra, we get

$$
\begin{aligned}
\Delta_{I}=\frac{1}{1-f}((c+1-f) \lg (c+1-f)-c \lg c \\
\quad-(f c+1-f) \lg (f c+1-f)+f c \lg (f c)) .
\end{aligned}
$$

The expressions $\Delta_{U}$ and $\Delta_{L}$ are used to bound the error in approximating $S_{U}$ by $I_{U}$ and $S_{L}$ by $I_{L}$ respectively.

$$
\begin{aligned}
& \Delta_{U}= \begin{cases}\lg \left(1+\frac{c}{q A}\right)-\lg \left(1+\frac{c}{p A+1}\right) & \text { if } p>q-1 / A \\
0 & \text { if } p \leq q-1 / A\end{cases} \\
& \Delta_{L}= \begin{cases}\lg \left(1+\frac{c}{p A}\right)-\lg \left(1+\frac{c}{q A}\right) & \text { if } p<q ; \\
0 & \text { if } p \geq q .\end{cases}
\end{aligned}
$$

We need a simple lemma from integral calculus. 
Lemma 3 If $g(x)$ is monotone increasing, then

$$
\sum_{j=0}^{c-1} g(j)<\int_{0}^{c} g(x) d x .
$$

If $g(x)$ is monotone decreasing, then

$$
\sum_{j=0}^{c-1} g(j)<\int_{0}^{c} g(x) d x-g(c)+g(0) .
$$

Proof: The increasing case is obvious. In the decreasing case, the integral for any unit interval is greater than the value at the right end of the interval: $\int_{j}^{j+1} g(x) d x>$ $g(j+1)$. We obtain the lemma by adding $g(j)-g(j+1)$ to both sides and summing over $j$.

Lemma 4 The code length $l$ for all occurrences of a single symbol in a block in ED order is less than $I_{L}+\Delta_{I}+\Delta_{U}$.

Proof: We show that $l<S_{U} \leq I_{U}+\Delta_{U}=I_{L}+\Delta_{I}+\Delta_{U}$. Since $S_{U}$ represents the code length for the symbol if all occurrences of the symbol come as late as possible in an ED order, we have $l<S_{U}$. If $p<q-1 / A$, then $-\lg p_{E}(x)$ is monotone increasing, so by Lemma 3 we have $S_{U}<I_{U}$. If $p>q-1 / A$, then $-\lg p_{E}(x)$ is monotone decreasing, so by Lemma 3 we have $S_{U}<I_{U}+\lg p_{E}(c)-\lg p_{E}(0)=I_{U}+\Delta_{U}$. If $p=q-1 / A$, then $S_{U}=I_{U}$. In all three cases, $S_{U} \leq I_{U}+\Delta_{U}$. From the definition of $\Delta_{I}, I_{U}=I_{L}+\Delta_{I}$.

We now relate $I_{L}$ to the entropy of the beginning and ending probability distributions. We write $I_{L}(i)$ to differentiate the values of $I_{L}$ evaluated for different symbols $a_{i}$. We define $H(R)$ and $H(Q)$ to be the entropies associated with probability distributions $R=\left\langle r_{1}, r_{2}, \ldots, r_{n}\right\rangle$ and $Q=\left\langle q_{1}, q_{2}, \ldots, q_{n}\right\rangle$ respectively; that is,

$$
\begin{aligned}
& H(R)=-\sum_{i=1}^{n} r_{i} \lg r_{i} \\
& H(Q)=-\sum_{i=1}^{n} q_{i} \lg q_{i} .
\end{aligned}
$$

Lemma 5 Let $L_{H}=B\left(\frac{1}{1-f} H(R)-\frac{f}{1-f} H(Q)\right)$. Then $L_{H}=\sum_{i=1}^{k} I_{L}(i)$.

Proof: By appropriate substitutions, we have

$$
\begin{aligned}
\sum_{i=1}^{k} I_{L}(i)= & \sum_{i=1}^{k} B\left(\frac{1}{1-f}\left(-r_{i} \lg r_{i}\right)-\frac{f}{1-f}\left(-q_{i} \lg q_{i}\right)\right) \\
& +\sum_{i=1}^{k} B\left(\frac{f}{(1-f)^{2}}\left(q_{i}-r_{i}\right)\right)
\end{aligned}
$$


The last sum is 0 because $Q$ and $R$ are probability distributions, so $\sum_{i=1}^{k} q_{i}=$ $\sum_{i=1}^{k} r_{i}=1$. The lemma follows from the definition of $H(\cdot)$.

Finally we bound the per-block error.

Lemma 6 Let $L_{j}$ be the compressed length of block $j$. Then

$$
L_{j} \leq B\left(\frac{1}{1-f} H(R)-\frac{f}{1-f} H(Q)\right)+k \lg \frac{B}{k}+O\left(\frac{k^{2}}{B}\right) .
$$

Proof: From Lemmas 4 and 5, we have $L_{j} \leq L_{H}+\sum_{i=1}^{k}\left(\Delta_{I}+\Delta_{U}\right)$. A scaling factor $f$ of $1 / 2$ implies that $A=B$. Asymptotics under this condition give

$$
\Delta_{I}+\Delta_{U}= \begin{cases}1-O(1 / c) & \text { if } p \leq q-1 / A \\ \lg (c / s)+O(s / c) & \text { if } p>q-1 / A\end{cases}
$$

The $\operatorname{sum} \sum_{i=1}^{k}\left(\Delta_{I}+\Delta_{U}\right)$ is maximized when as many symbols as possible have large $c$ and small $s$; the sum's largest possible value cannot exceed its value when $s=m$ and $c=B / k$ for all $k$ symbols, in which case $\Delta_{I}+\Delta_{U}=\lg (B / \mathrm{km})+O(\mathrm{~km} / B)$. We obtain the result by setting $m=1$ and summing over the symbols.

The proof of the upper bound in Theorem 1 follows from Lemma 6 by summing over all blocks, noting that $b=t / B$. (We are neglecting any special effects of a longer first block or shorter last block.) There is much cancellation because $H(R)$ of one block is $H(Q)$ of the next.

\subsubsection{Proof of the lower bound}

The proof of the lower bound of Theorem 1 is similar to that of the upper bound. In this proof of the lower bound we append a prime to the label of each definition and lemma to show the correspondence with the definition and lemmas used in the proof of the upper bound.

Definition $2^{\prime}$ A block of length $B$ containing $c_{1}, c_{2}, \ldots, c_{k}$ occurrences of symbols $a_{1}, a_{2}, \ldots, a_{k}$, respectively, has the reverse $E D$ property if for each symbol $a_{i}$ and for all $m, 1 \leq m \leq c_{i}$, the symbol occurs for the $m$ th time not before position $(m-1) B / c_{i}$.

Lemma $2^{\prime}$ Every distribution of symbol counts has an order with the reverse ED property.

Proof: By Lemma 2 there is always an order with the ED property. Such an order, when reversed, has the reverse ED property. 
Lemma $3^{\prime}$ If $g(x)$ is monotone decreasing, then

$$
\sum_{j=0}^{c-1} g(j)>\int_{0}^{c} g(x) d x .
$$

If $g(x)$ is monotone increasing, then

$$
\sum_{j=0}^{c-1} g(j)>\int_{0}^{c} g(x) d x-g(c)+g(0) .
$$

Proof: Similar to that of Lemma 3.

Lemma $4^{\prime}$ The code length $l$ for all occurrences of a single symbol in a block in reverse ED order is greater than $I_{L}-\Delta_{L}$.

Proof: We show that $l>S_{L} \geq I_{L}-\Delta_{L}$. Since $I_{L}$ represents the code length for the symbol if all occurrences of the symbol come as early as possible in a reverse ED order, we have $l>S_{L}$. If $p>q$, then $-\lg p_{B}(x)$ is monotone decreasing, so by Lemma $3^{\prime}$ we have $S_{L}>I_{L}$. If $p<q$, then $-\lg p_{B}(x)$ is monotone increasing, so by Lemma $3^{\prime}$ we have $S_{L}>I_{L}+\lg p_{B}(c)-\lg p_{B}(0)=I_{L}-\Delta_{L}$. If $p=q$, then $S_{L}=I_{L}$. In all three cases, we have $S_{L} \geq I_{L}-\Delta_{L}$.

Lemma $6^{\prime}$ Let $L_{j}$ be the compressed length of block $j$. Then

$$
L_{j} \geq B\left(\frac{1}{1-f} H(R)-\frac{f}{1-f} H(Q)\right)-k .
$$

Proof: From Lemmas $4^{\prime}$ and $5, L_{j} \geq L_{H}-\sum_{i=1}^{k} \Delta_{L}(i)$. Asymptotics when $f=1 / 2$ give $\Delta_{L}=1-O(\mathrm{c} / \mathrm{s})$, which has maximum value 1 , so the sum is at most $k$.

The proof of the lower bound in Theorem 1 follows from Lemma $6^{\prime}$ by summing over all blocks, noting that $b=t / B$.

\subsubsection{Non-scaling corollary}

By letting $B=t, f=n /(t+n)$, and $m=1$ in Lemmas 6 and $6^{\prime}$, we obtain the code length without scaling:

Corollary 1 When we do not scale at all, the code length $L_{N S}$ satisfies:

$$
t H_{\text {final }}+n\left(H_{\text {final }}-H_{0}\right)-k<L_{N S}<t H_{\text {final }}+n\left(H_{\text {final }}-H_{0}\right)+k \lg (t / k) .
$$

We can get important insights by contrasting upper bounds in this corollary and Theorem 1. Scaling will bring about a shorter encoding by tracking the block-byblock entropies rather than matching a single entropy for the entire file, but when we forgo scaling the overhead is less, proportional to $\lg t$ instead of to $t$. Scaling will do worst on a homogeneously distributed file, but even then the overhead will increase the code length by only about $(k / B) \lg (B / k)$ bits per input symbol, less than 0.1 bit per symbol for a typical file. We conclude that the benefits of scaling usually outweigh the minor inefficiencies it sometimes introduces. 


\section{$2.2 \quad$ Application to Higher Order Models}

We now extend our results to higher order models. Cleary and Witten [6] present a practical adaptive method called prediction by partial matching (or $P P M$ ) in which they maintain models of various orders. At each point they use the highest-order model in which the symbol has occurred in the current context, with a special escape symbol to indicate the need to drop to a lower order. (Because most contexts occur only a few times with only a few different symbols, assigning an initial weight of 1 to each alphabet symbol as we did in Section 1.1 is an unsatisfactory solution to the zero-frequency problem in higher-order models. Doing so would give too much weight to symbols that never occur.) See [2] or [3] for a detailed description of the PPM method. Witten, Cleary, Moffat, and Bell have proposed at least five methods for estimating the probability of the escape symbol [6,19,33], and Arps et al. [1] give two more. All of the methods give approximately the same compression; PPMB [6] is the most readily analyzed.

In PPMB, in each context the escape event is treated as a separate symbol with its own weight and probability; the first occurrence of an ordinary symbol is not counted and the first two occurrences are coded as escapes. Treating the escape event as a normal symbol, we can apply the results of Section 2 if we make adjustments for the first two occurrences of each symbol, since in PPMB the code length is independent of the order of the symbols.

In the block in which a given symbol occurs for the first time, we can take the occurrences to be evenly distributed in the sense of Definition 2, with symbol weights (numerators) running from 1 to $c$ and occurrence positions (denominators) running from $A+B / c$ to $A+B$. If this were coded in the normal way, the code length would be bounded above by Lemmas 4 and 5. Since the mechanism of PPMB excludes the last two numerators, $c-1$ and $c$, and the first two denominators, $A+B / c$ and $A+2 B / c$, the code length from the approximation must be adjusted by adding $L_{\text {adjustment: }}$ :

$$
\begin{aligned}
L_{\text {adjustment }}= & L_{\text {actual }}-L_{\text {estimated }} \\
= & \lg \frac{(c-1) c}{(A+B / c)(A+2 B / c)} \\
= & 2 \lg c-2 \lg A+\lg \left(1-\frac{1}{c}\right)-\lg \left(1+\frac{1 / f-1}{c}\right) \\
& \quad-\lg \left(1+2 \frac{1 / f-1}{c}\right) .
\end{aligned}
$$

We must also adjust the entropy: the actual value of the initial probability $q$ is 0 instead of $1 / A$, and the actual value of the final probability $r$ is $(c-1) /(A+B)$ instead of $(c+1) /(A+B)$. For convenience we denote the entropy term $B\left(\frac{1}{1-f} H(R)-\frac{f}{1-f} H(Q)\right)$ by $h$. We define and compute the adjustment:

$$
h_{\text {adjustment }}=h_{\text {actual }}-h_{\text {estimated }}
$$




$$
=c \lg \left(1+\frac{2}{c-1}\right)+2 \lg c-\lg A+2 \lg f+\lg \left(1-\frac{1}{c^{2}}\right) .
$$

The code length is then given by

$$
\begin{aligned}
L_{\text {actual }}= & h_{\text {actual }}+\left(L_{\text {adjustment }}-h_{\text {adjustment }}\right)+\text { small terms } \\
= & h_{\text {actual }}-c \lg \left(1+\frac{2}{c-1}\right)-\lg A-2 \lg f-\lg \left(1+\frac{1}{c}\right) \\
& -\lg \left(1+\frac{1 / f-1}{c}\right)-\lg \left(1+2 \frac{1 / f-1}{c}\right) \\
& + \text { small terms. }
\end{aligned}
$$

The net adjustment is always negative if $f=1 / 2$. We can let $f=1 / 2$ if we neglect the effect of the time before the first scaling in each context.

Now we can extend Theorem 1 to the PPMB model with scaling, using $X$ subscripts in the natural way to restrict quantities to context $X$ :

Theorem 2 When we use PPMB with scaling, the code length $L$ is bounded by

$$
L<\sum_{\text {contexts } X}\left(B\left(\sum_{m=1}^{b_{X}} H_{X, m}+H_{X, b}-H_{X, 0}\right)+O\left(\frac{k_{X} t_{X} \lg B}{B}\right)\right) .
$$

Proof: The proof follows from the discussion above.

This theorem does not readily estimate the code length of a file in a direct way. However, it does show that the code length of a file coded using a high-order Markov model with scaling can be expressed using the weighted entropy formulation introduced in Section 2. In particular, the code length for each context is expressed directly in terms of the weighted entropies for that context.

\section{Coding Effects}

In this section we prove analytically that coding effects (as distinguished from modeling effects) are insignificant, and hence that our assumption of exact coding is appropriate. Empirical evidence that the coding effects are negligible appears in $[2$, 34].

\subsection{Rounding counts to integers}

In Section 2 we analyzed the modeling effect of periodic scaling; here we analyze the coding effect. Witten, Neal, and Cleary scale the counts to avoid register overflow, and to prevent any count from falling to 0 , they round fractional counts to the next higher integer. This gives more weight to symbols whose count happens to be odd when scaling occurs. 
Theorem 3 Rounding counts up to the next higher integer increases the code length for the file by no more than $n / 2 B$ bits per input symbol.

Proof: Each symbol whose weight is rounded up causes the denominators of all probabilities in the next block to be too large, by 1/2. If $r$ is the number of symbols subject to roundup, $r / 2$ of the denominators in computing the coding interval will be approximately $T$ instead of $T / 2$, each adding one bit to the code length of the block, so the block's code length will be $r / 2$ bits longer. The effect for the entire file $(t / B$ blocks) is $r t / 2 B$ bits, or, since $r \leq n$, at most $n / 2 B$ bits per input symbol.

This effect is typically 0.02 bit or less per input symbol.

\subsection{Using integer arithmetic}

Witten, Neal, and Cleary use integers from a large fixed range, typically $[0,8 B]$, instead of using exact rational arithmetic, and they transmit encoded bits as soon as they know them instead of waiting until the entire string has been encoded, scaling up the range to represent only that half of the original range whose identity has not yet been transmitted. The result is nearly the same compression efficiency as with exact rational arithmetic.

As is apparent from the following description of the coding section of the WittenNeal-Cleary algorithm, scaling up the range is only approximate:

1. We select a subrange of the current interval [low, high] whose length within $[l o w, h i g h]$ is proportional to $p$. The new integer values of low and low are obtained by truncating the results of the exact calculation.

2. We repeat the following steps as many times as possible:

(a) If the new subrange is not entirely in the lower, middle, or upper half of the full range of values, we return.

(b) If the new subrange is in the lower or upper half, we output 0 or 1 respectively, plus any bits left over from previous symbols. If the subrange is entirely in the middle half, we keep track of this fact for future output.

(c) We scale the subrange up:

i. We shift the subrange to ignore the part of the full range in which the subrange is known not to lie.

ii. We double both low and high and add 1 to high.

In this algorithm, any roundoff error in selecting the first subrange will propagate through the entire scaling up process. In the worst case, a symbol with a count of 1 could result in a subrange of length 1 , even though the unrounded subrange size might be just below 2. In effect, this would assign a symbol probability of only half 
the correct value, resulting in a code length one bit too long. In practice, however, the code length error in one symbol is seldom anywhere near that large, and because the errors can be of either sign and have an approximately symmetrical distribution with mean 0 , the average error is usually very small. Witten, Neal, and Cleary empirically estimate it at $10^{-4}$ bit per input symbol.

In order to get a rigorous bound on the compression loss, we analyze a new algorithm that maintains full precision when scaling up the range. Instead of adding 1 to high at each step, we add either 0 or 1 to low, and independently we add 1 or 2 to high, the choice in each case being based on the fractional bits of the exact results of the initial subrange selection. The resulting code length may be longer than that of exact arithmetic coding, but by a tiny amount, as shown in the following theorem:

Theorem 4 When we use the high precision algorithm for scaling up the subrange, the code length does not exceed the ideal code length $-\lg p$ by more than $1 /(2 B \ln 2)$ bits per input symbol.

Proof: After the scaling up process, the smallest possible subinterval size is $2 \mathrm{~B}$. As a result of rounding while scaling up, low can never be too high, and high can be too low by as much as 1 , so the subinterval can be too short by as much as 1 . (It can also be too long by as much as 1.) An interval too short by 1 can be too short by a factor of as much as $(2 B-1) / 2 B$, which corresponds to a code length increase of $-\lg ((B-1 / 2) / B) \sim 1 /(2 B \ln 2)$.

A loss of $1 /(2 B \ln 2)$ bits per input symbol is negligible, about $10^{-4}$ bit per symbol for typical parameters. In practice the high precision algorithm gives exactly the same compression as the algorithm of Witten, Neal, and Cleary, but its compression loss is provably small.

\subsection{Encoding end-of-file}

The end of the file must be explicitly indicated when we use arithmetic coding. The extra code length required is typically very small and is often ignored; for completeness, we provide a brief analysis of the end-of-file effect.

Witten, Neal, and Cleary introduce a special low-weight symbol in addition to the normal alphabet symbols; it is encoded once, at the end of the file. In the following theorem we bound the cost of identifying end-of-file by this method:

Theorem 5 The use of a special end-of-file symbol results in additional code length of less than $t /(B \ln 2)+\lg B+10$ bits.

Proof: The cost has four components:

- at most $\lg B+1$ bits to encode the end-of-file symbol (since its probability must be at least as large as the smallest possible probability, 1/2B) 
- fewer than $t /(B \ln 2)$ bits in wasted code space to allow end-of-file at any point (each probability can be reduced by a factor of between $(2 B-1) / 2 B$ and $(B-1) / B$, resulting in a loss of between $-\lg ((2 B-1) / 2 B) \approx 1 /(2 B \ln 2)$ and $-\lg ((B-1) / B) \approx 1 /(B \ln 2)$ bits per symbol $)$

- two disambiguating bits after the end-of-file symbol

- up to seven bits to fill the last byte.

An alternative, transmitting the length of the original file before its encoding, reduces the cost to between $\lg t$ and $2 \lg t$ bits by using an appropriate encoding of integers [11,31,32], but requires the file length to be known before encoding can begin.

The end-of-file cost using either of these methods is negligible for a typical file, less than 0.01 bit per input symbol.

\section{Conclusion}

Using our notion of weighted entropy, we have precisely characterized the tradeoff between the overhead associated with scaling and the saving that it can realize by exploiting locality of reference. The largest code length savings come from more sophisticated (higher order) models such as PPMB, and our scaling analysis extends accordingly. We have also proven that the computational effects on the code length in practical arithmetic coding implementations are small, so we can treat practical arithmetic coders as though they were exact coders.

Another important consideration in making arithmetic coding practical, which we do not address in this article, is the speed at which the current interval can be updated. In the basic algorithm outlined in Section 1 and in the work of Witten, Neal, and Cleary, up to two multiplications and one division are needed for each symbol encoded. Work by Rissanen, Langdon, Mohiuddin, and others at IBM [5,16,18,22,27] eliminates the division altogether and focuses on approximating the multiplication by combinations of additions and shifts. In [13] we present an alternative approach in which we approximate an arithmetic coder by a finite state automaton with a small number of states. Since the arithmetic computations are effectively stored in the state tables, coding can proceed quickly using only table lookups.

Acknowledgement. We wish to thank Prof. Martin Cohn for helping us to uncover a small mistake in the analysis of the effect of using integer arithmetic. 


\section{References}

[1] R. B. Arps, G. G. Langdon \& J. J. Rissanen, "Method for Adaptively Initializing a Source Model for Symbol Encoding," IBM Technical Disclosure Bulletin 26 (May 1984), 6292-6294.

[2] T. C. Bell, J. G. Cleary \& I. H. Witten, Text Compression, Prentice-Hall, Englewood Cliffs, NJ, 1990.

[3] T. C. Bell, I. H. Witten \& J. G. Cleary, "Modeling for Text Compression," Comput. Surveys 21 (Dec. 1989), 557-591.

[4] J. L. Bentley, D. D. Sleator, R. E. Tarjan \& V. K. Wei, “A Locally Adaptive Data Compression Scheme," Comm. ACM 29 (Apr. 1986), 320-330.

[5] D. Chevion, E. D. Karnin \& E. Walach, "High Efficiency, Multiplication Free Approximation of Arithmetic Coding," in Proc. Data Compression Conference, J. A. Storer \& J. H. Reif, eds., Snowbird, Utah, Apr. 8-11, 1991, 43-52.

[6] J. G. Cleary \& I. H. Witten, "Data Compression Using Adaptive Coding and Partial String Matching," IEEE Trans. Comm. COM-32 (Apr. 1984), 396-402.

[7] J. G. Cleary \& I. H. Witten, "A Comparison of Enumerative and Adaptive Codes," IEEE Trans. Inform. Theory IT-30 (Mar. 1984), 306-315.

[8] G. V. Cormack \& R. N. Horspool, "Data Compression Using Dynamic Markov Modelling," Computer Journal 30 (Dec. 1987), 541-550.

[9] G. V. Cormack \& R. N. Horspool, “Algorithms for Adaptive Huffman Codes," Inform. Process. Lett. 18(Mar. 1984), 159-165.

[10] P. Elias, "Interval and Recency Rank Source Coding: Two On-line Adaptive Variable Length Schemes," IEEE Trans. Inform. Theory IT-33(Jan. 1987), 3-10.

[11] P. Elias, "Universal Codeword Sets and Representations of Integers," IEEE Trans. Inform. Theory IT-21 (Mar. 1975), 194-203.

[12] A. S. Fraenkel \& S. T. Klein, "Robust Universal Complete Codes as Alternatives to Huffman Codes," Dept. of Applied Mathematics, The Weizmann Institute of Science, Technical Report, Rehovot, Israel, 1985.

[13] P. G. Howard \& J. S. Vitter, "Practical Implementations of Arithmetic Coding," in Image and Text Compression, J. A. Storer, ed., Kluwer Academic Publishers, Norwell, MA, 1992, 85-112.

[14] D. A. Huffman, "A Method for the Construction of Minimum Redundancy Codes," Proceedings of the Institute of Radio Engineers 40 (1952), 1098-1101.

[15] D. E. Knuth, "Dynamic Huffman Coding,” J. Algorithms6 (June 1985), 163-180. 
[16] G. G. Langdon, "Probabilistic and Q-Coder Algorithms for Binary Source Adaptation," in Proc. Data Compression Conference, J. A. Storer \& J. H. Reif, eds., Snowbird, Utah, Apr. 8-11, 1991, 13-22.

[17] G. G. Langdon, "An Introduction to Arithmetic Coding," IBM J. Res. Develop. 28 (Mar. 1984), 135-149.

[18] G. G. Langdon \& J. Rissanen, "Compression of Black-White Images with Arithmetic Coding," IEEE Trans. Comm. COM-29 (1981), 858-867.

[19] A. M. Moffat, "Implementing the PPM Data Compression Scheme," IEEE Trans. Comm. COM-38 (Nov. 1990), 1917-1921.

[20] K. Mohiuddin, J. J. Rissanen \& M. Wax, “Adaptive Model for Nonstationary Sources," IBM Technical Disclosure Bulletin 28 (Apr. 1986), 4798-4800.

[21] R. Pasco, "Source Coding Algorithms for Fast Data Compression," Stanford Univ., Ph.D. Thesis, 1976.

[22] W. B. Pennebaker, J. L. Mitchell, G. G. Langdon \& R. B. Arps, "An Overview of the Basic Principles of the Q-Coder Adaptive Binary Arithmetic Coder," IBM J. Res. Develop. 32 (Nov. 1988), 717-726.

[23] J. Rissanen, "Stochastic Complexity and Modeling," Ann. Statist.14 (1986), 10801100 .

[24] J. Rissanen, "Stochastic Complexity," J. Roy. Statist. Soc. Ser. B 49(1987), 223239, 253-265.

[25] J. Rissanen, "Universal Coding, Information, Prediction, and Estimation," IEEE Trans. Inform. Theory IT-30 (July 1984), 629-636.

[26] J. J. Rissanen, "Generalized Kraft Inequality and Arithmetic Coding," IBM J. Res. Develop. 20 (May 1976), 198-203.

[27] J. J. Rissanen \& K. M. Mohiuddin, “A Multiplication-Free Multialphabet Arithmetic Code," IEEE Trans. Comm. 37 (Feb. 1989), 93-98.

[28] F. Rubin, "Arithmetic Stream Coding Using Fixed Precision Registers," IEEE Trans. Inform. Theory IT-25 (Nov. 1979), 672-675.

[29] B. Y. Ryabko, "Data Compression by Means of a Book Stack," Problemy Peredachi Informatsii $16(1980)$.

[30] C. E. Shannon, "A Mathematical Theory of Communication," Bell Syst. Tech. J. 27 (July 1948), 398-403.

[31] R. G. Stone, "On Encoding of Commas Between Strings," Comm. ACM 22 (May 1979), 310-311.

[32] M. Wang, “Almost Asymptotically Optimal Flag Encoding of the Integers," IEEE Trans. Inform. Theory IT-34 (Mar. 1988), 324-326. 
[33] I. H. Witten \& T. C. Bell, "The Zero Frequency Problem: Estimating the Probabilities of Novel Events in Adaptive Text Compression," IEEE Trans. Inform. Theory IT-37 (July 1991), 1085-1094.

[34] I. H. Witten, R. M. Neal \& J. G. Cleary, “Arithmetic Coding for Data Compression," Comm. ACM 30 (June 1987), 520-540. 